\section{Agenda building. Revisión de la literatura sobre el proceso de construcción de la agenda mediática}

doi:10.11144/Javeriana.syp36-70.abrl

Agenda Building. A Literature Review of the

Construction Process of the Media Agenda

Agenda building. Revisão da literatura sobre o processo

de construção da agenda mediática
Recibido: 2 de julio de 2016 Aceptado: 13 de diciembre de 2016 Disponible en línea: 30 de mayo de 2017

Submission Date: July $2^{\text {nd }}, 2016$ Acceptance Date: December $13^{\text {th, }} 2016$ Available Online: May $30^{\text {th }}, 2017$

\section{Origen del artículo}

El presente artículo se origina a partir de una revisión de la literatura de la Teoría de la Agenda Setting realizada entre 2013 y 2015. La investigación, denominada $E l$ poder de la agenda. Política, medios y público fue adelantada bajo la tutela del Consejo Nacional de Investigaciones Científicas y Técnicas (Conicet) y la Universidad Nacional de Quilmes, y fue financiada por el Conicet. 


\section{Resumen}

Este artículo propone una revisión bibliográfica que permite reflexionar acerca de los factores endógenos y exógenos que intervienen, con mayor o menor efectividad, en el proceso de construcción de la agenda mediática. El análisis se centra en el vínculo que hay entre los medios y los tomadores de decisiones; en la activa participación de los medios en el campo político; en la capacidad de las fuentes de información de instalar la agenda de los quiénes (Charron, 1998), y en los niveles de influencia que distintos actores ejercen sobre la agenda mediática, instalando u ocultando un asunto, y promoviendo una determinada definición de la realidad.

Palabras clave: agenda building; medios; tomadores de decisiones; fuentes de información; actores sociales

\section{Abstract}

This article proposes a literature review that allows a critical reflection on endogenous and exogenous factors involved, with varying degrees of effectiveness, on the construction process of the media agenda. The analysis focuses on the link there is between the media and decision makers; on the active participation of the media in the political field; on the capacity of sources of information to install the agenda of whom (Charron, 1998), and on the levels of influence that different actors exert on the media agenda, installing or hiding an issue, and promoting a certain definition of reality.

Keywords: agenda building; media; decision makers; sources of information; social actors

\section{Resumo}

Este artigo propõe uma revisão bibliográfica permitindo refletir acerca dos fatores endógenos e exógenos envolvidos, com maior ou menor efetividade, no processo de construção da agenda mediática. A análise centra-se na ligação entre mídia e tomadores de decisão; na ativa participação dos meios na arena política; na capacidade das fontes de informação para fixar a agenda dos quem (Charron, 1998), e nos níveis de influência que diferentes atores exercem sobre a agenda mediática, instalando u ocultando um assunto e a promover uma certa definição da realidade.

Palavras-chave: agenda building; meios; tomadores de decisões; fontes de informação; atores sociais 
Natalia Aruguete

\section{Agenda building. Revisión de la literatura sobre el proceso de construcción de la agenda mediática}

Introducción

A los medios se les suele atribuir un rol predominantemente pasivo: el de agencias de información neutrales que proveen un foro para que otros actores expresen sus ideas. Pero no son neutrales; son actores políticos con intereses y metas genuinos que interactúan con otros, en un proceso de conflictividad y negociación permanente que involucra la generación de sentidos públicos. Su actuación, entonces, será coherente con los rasgos de la sociedad en la que se inserten y, dentro de esta, con el vínculo que establezcan con quienes participen de esa comunidad.

El rol activo que los medios desempeñan en el proceso de toma de decisiones políticas (Cohen, 1993), sin embargo, no les garantiza que su postura frente a ciertos temas tenga impacto en el ámbito político. En consecuencia, el interés de este trabajo reside en problematizar, mediante una revisión de la literatura relativa al proceso de agenda building, el tipo de relación que los medios entablan con aquellos actores — las fuentes de información, los líderes políticos, los otros medios de comunicación, entre otros- que contribuyen a configurar sus menús de temas.

\section{La agenda building}

Desde los estudios realizados por Robert Park y Walter Lippmann, durante cuatro décadas (19301970) hubo pocos intentos sistemáticos de estudiar la posición del emisor dentro de una red social que seleccionaba y rechazaba información en función de una serie de factores determinados (Cervantes Barba, 2001). En la década de 1950, Warren Breed indagó desde distintas vertientes por las prácticas profesionales del periodismo en su ámbito laboral, mientras que White (1950) se enfocó en los factores que funcionaban como guardabarreras de la información que ingresaba a las instituciones mediáticas. Pero entre mediados de los años 70 y principios de los 80 resurgieron iniciativas centradas en el análisis de los productores de la información desde una perspectiva sociológica (Gitlin, 1986; Kunczik, 1991; Schlesinger, 1992; Tuchman, 1983; Weaver y Gray, 1982; Wright, 1959). Por aquella época, diversos investigadores comenzaron a estudiar el tipo de interacción que se daba entre la agenda política y la mediática. Nació así la llamada agenda building.

La agenda building se centra en el proceso de construcción de las noticias y los elementos que lo 
influyen. ¿Cuándo surgen los estudios de agenda building? Desde una perspectiva cronológica, la agenda building se ubica en la denominada cuarta fase de la Teoría de la Agenda Setting (McCombs, 2006). La primera fase se inició con el estudio seminal realizado en el año 1968, cuando los investigadores Maxwell McCombs y Donald Shaw confirmaron que los medios tenían efectos cognitivos en los votantes. La segunda fase introdujo el análisis de las condiciones contingentes que mediaban la relación entre medios y públicos. La tercera fase abrió paso a la llamada attribute agenda setting, con la cual se modificó el tipo de influencia mediática que era contemplado, que pasó de ser cognitivo a ser actitudinal. En medio de esta evolución, la cuarta fase supuso un giro copernicano de tal perspectiva teórica. Del análisis de los efectos mediáticos en la agenda pública, se pasó a una exploración que se desenvuelve sobre el proceso mismo de construcción de la agenda de los medios de comunicación. La pregunta de investigación apuntó en esta etapa hacia los factores que dejan huellas y moldean las coberturas noticiosas. En otras palabras, la agenda mediática cedió su rol de factor explicativo de la percepción de las audiencias y se convirtió en una variable dependiente, influida por un proceso complejo en el que intervienen limitaciones institucionales (Sigal, 1973), valores noticiosos (Golding, 1981), hábitos profesionales, valores asociados al rol social del periodista que son internalizados individual o colectivamente (Gans, 2004), y rutinas de trabajo (Shoemaker y Reese, 1996; Tuchman, 1972).

La emergencia de la agenda periodística como objeto de análisis se explica por la confluencia de una "red compleja de factores endógenos y exógenos, estructurales y contextuales, que dan forma a los contenidos noticiosos" (Brandenburg, 2002, p. 39). ${ }^{1}$ A comienzos de la década de 1980, Kurt Lang y Gladys Lang (1981) notaron que los investigadores de la comunicación omitían preguntarse por cómo se forma la agenda pública. Hay poco o ningún "reconocimiento del proceso a través del cual se construyen las agendas o mediante el cual un objeto que es alcanzado por la atención pública [...] da lugar a un tema político" (p. 448). Si bien subyace un rol preponderante de las instituciones informativas en el proceso de construcción de la agenda, estas no dictan por sí mismas la agenda pública. La advertencia de estos investigadores es clave para los estudios de agenda setting, en la medida en que propone un cambio en la mirada arrojada sobre la relación $-\mathrm{y}$, por ende, la correlación de fuerzas - que se da entre 
tres agendas: la agenda mediática, la agenda política y la agenda pública. De ahí la decisión de autores como Charron (1998) de reemplazar el término establecimiento por el de construcción para designar al "proceso colectivo de elaboración de la agenda" (pp. 79-80), que implica cierta reciprocidad entre los medios, los tomadores de decisiones y el público.

El matrimonio Lang propone un modelo de cuatro pasos en el que quedan englobados los procesos de agenda setting y de agenda building. Primero, los medios destacan ciertos eventos, actividades, grupos o personalidades. Luego, los elementos de un conflicto se combinan en un marco común, subrayando o escondiendo ciertos aspectos del objeto. En tercer lugar, el tema en cuestión se asocia a símbolos secundarios, convirtiéndose en parte de un paisaje político reconocido. Finalmente, los portavoces juegan un papel importante en la promoción de los temas y los símbolos. La primera etapa corresponde al establecimiento de la agenda. La segunda, tercera y cuarta, a la construcción de la agenda (citado por Sádaba, 2008).

Figura 1. Las capas de la cebolla

\section{Las capas de la cebolla}

¿Quién establece la agenda de los medios? Los determinantes del proceso de producción de las agendas mediáticas quedaron plasmados en la metáfora de las capas de la cebolla (McCombs, 1992).

En un recorrido de afuera hacia adentro, en la capa exterior se ubican los hacedores principales de la información externos a la institución informativa, como presidentes, partidos políticos, instituciones oficiales $u$ oficinas de relaciones públicas de las empresas. En segundo lugar, se encuentran los propios medios de comunicación, que se influyen unos a otros, evidenciando el poder existente entre ellos para fijar la agenda (intermedia agenda setting). En tercer lugar, los soportes técnicos se convierten en condicionantes significativos de la producción mediática. La cuarta capa incluye las restricciones impuestas por la cultura organizacional. La quinta remite por su parte a las preferencias individuales de los periodistas y sus posiciones ideológicas. Finalmente, en el corazón de la cebolla se ubican los estilos o géneros

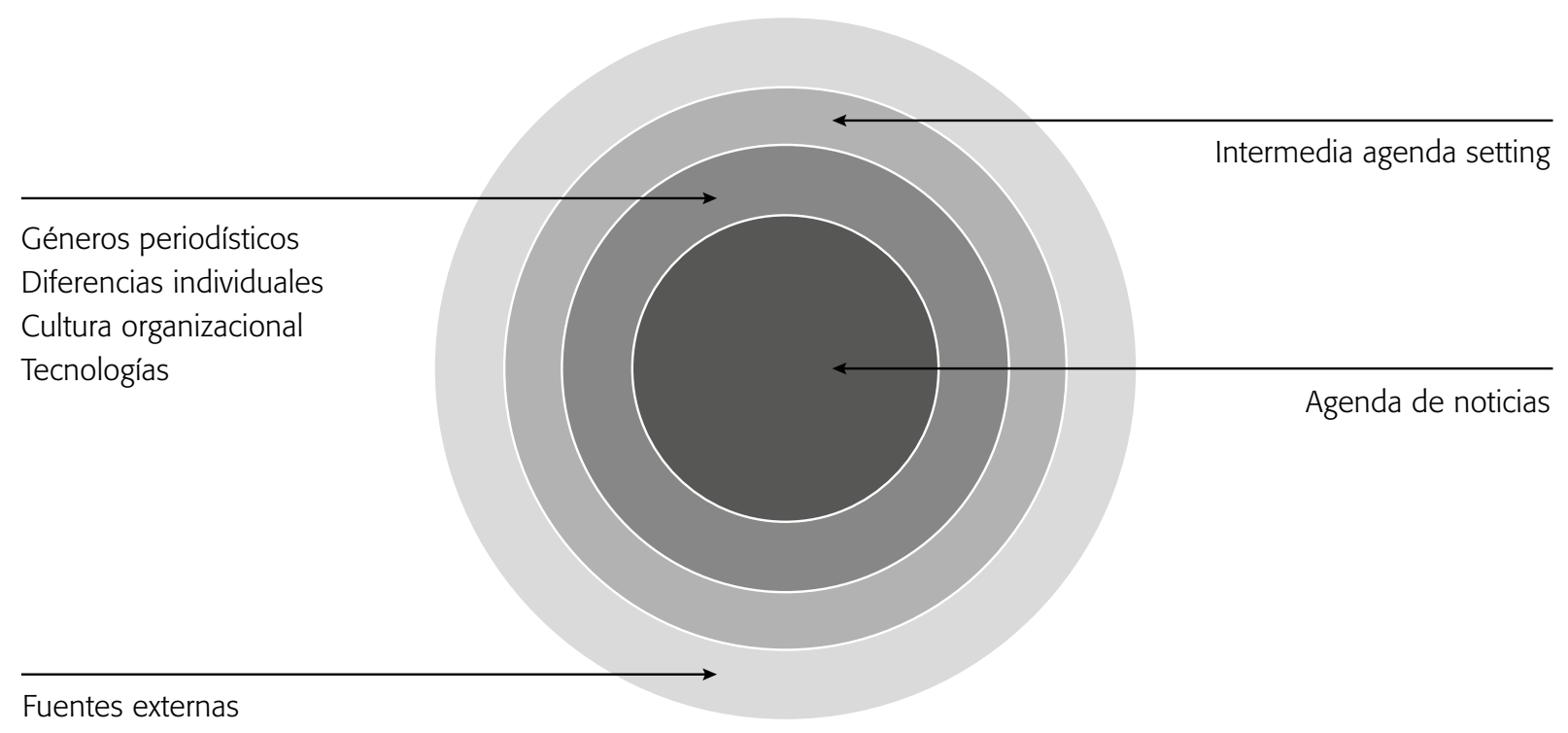


periodísticos que hacen a la labor profesional. En los próximos apartados revisaremos la bibliografía relativa a algunas de estas capas.

\section{Los hacedores de noticias (agenda setters)}

En el proceso de formación de la agenda mediática, la capa externa de la cebolla aloja a los hacedores de noticias, término con el cual se designa a presidentes u otro tipo de funcionarios públicos, así como a dirigentes políticos de otras índoles, aun cuando no pertenezcan a la estructura de un gobierno y corporaciones sindicales o económicas, entre otros organismos. El presidente de los Estados Unidos suele ser identificado como uno de los principales agenda setters, estatus que lo ubica en una posición más que estratégica. Su discurso ante el Congreso sobre el Estado de la Unión ha sido un objeto de estudio muy recurrente en el examen del grado de influencia del poder político sobre la agenda mediática, por ser un reporte multifacético que suele tocar una importante variedad de asuntos nacionales y, más aún, por tratarse de un intento explícito del presidente de impactar notablemente en la percepción que la opinión pública tiene de la relevancia de los temas (Gilberg, Eyal, McCombs y Nicholas, 1991).

De los diversos estudios elaborados sobre esta interacción, no surge un consenso que indique en qué sentido viaja el efecto de la agenda. Por ejemplo, los temas priorizados en el discurso pronunciado por el presidente Richard Nixon ante el Parlamento en 1970 fueron posteriormente registrados en las coberturas de la NBC, el New York Times y el Washington Post (Wanta, Stephenson, Vanslyke Turk y McCombs, 1989). En cambio, Gilberg et al. (1991) llegaron a resultados opuestos en su análisis sobre el Estado de la Unión expuesto por el presidente James Carter en 1978: demostraron que la cobertura noticiosa tuvo efectos de agenda en las palabras del primer mandatario. Más aventurado, Wanta analizó la relación que se establece entre el presidente, los medios y el público, es decir: comparó los vínculos que hay entre el presidente y el público, y los medios y el público en cuanto a la relevancia otorgada a los temas, y encontró que ambas variables independientes - la palabra presidencial y la cobertura noticiosa - son predictores significativos de la percepción pública, aunque la influencia mediática pese más (Wanta, 1997, pp. 82-83).

Más allá del papel de agenda setter de la figura presidencial analizado hasta aquí, los resultados son contradictorios entre quienes asumen que los medios juegan un rol considerable en el aumento de la atención política hacia los asuntos (Soroka, 2002; Baumgartner, Bryan y Leech, 1997; Trumbo, 1996), y aquellos que observan un efecto limitado de estos sobre la agenda política (Herman, 1993; Livingston, 1997; Mermin, 1997).

En un intento de complejizar la comprensión del vínculo que hay entre medios y fuentes de información, Charron (1998, p. 74) observa que el modelo de la agenda building es insuficiente para estudiar la relación que se teje entre los medios y la fuente. Concretamente, trasladar el modelo inspirado en el vínculo existente entre los medios y el público para explicar el entramado del proceso de construcción de la agenda mediática conlleva problemas de traducción. El autor señala diversos aspectos de dicha limitación. Para empezar, se concede la influencia de un actor sobre la agenda noticiosa solo por el espacio que ocupa, sin considerar que dicho ascendiente puede deberse a la decisión de actores invisibles de no figurar o de hacerlo en contextos noticiosos de mayor estabilidad. Aquello involucra una segunda deficiencia analítica: la confusión de fuentes con actores, que deja vacante el espacio para un análisis real del impacto de unos sobre otros. Por su parte, Aruguete (2011, 2015) afirma que las fuentes provenientes del poder político, que cuentan con un alto grado de institucionalidad, acceden a la prensa en circunstancias más estables. En cambio, los más vulnerables, deslegitimados y sin amparo institucional, son objeto de una exposición poco cuidadosa y sus opiniones suelen aparecer en circunstancias noticiosas conflictivas y en situaciones dramáticas.

Los medios le importan a la política. Es algo inevitable. Están involucrados en el proceso 
de toma de decisiones e intervienen de distintas maneras, por ejemplo, dirigiendo la atención hacia determinados dominios públicos y ocultando otros (Soroka, Lawlor, Farnsworth y Young, 2012), y definiendo así el mapa político que el público atenderá como verdadero y que, como tal, puede tener consecuencias en determinadas decisiones políticas (Cohen, 1993). Ahora bien, el impacto mediático en la agenda política es contingente: depende del tipo de medio y los temas que se allí traten, al igual que de la agenda política que se organiza en función del período - electoral y no electoral- en el que se examine tal efecto (Walgrave y van Aels, 2006). En los próximos apartados, se revisan diversas hipótesis y hallazgos empíricos, atendiendo a los factores mencionados aquí, que por demás intervienen en este diálogo: los tipos de medios, los temas y el período estudiado.

\section{Los tipos de medios}

¿Todos los tipos de noticias tienen el mismo poder de influir en la agenda política? ¿Existen diferencias entre los medios a la hora de fijar los temas que luego serán considerados por los líderes políticos en sus decisiones? ¿Qué dinámica se da entre la agenda política y la agenda mediática, a partir de la irrupción de las redes sociales? Esas son algunas de las preguntas que guiarán el desarrollo de este apartado.

Los diarios nacionales suelen tener efectos distintos de los que tienen los periódicos locales y los medios audiovisuales. Bartels (1996) afirma que la influencia de The New York Times difiere de aquella que pueden ejercer los diarios locales que, a su vez, se diferencian de los programas televisivos. Más aún, la influencia política de ese gigante mediático se da indirectamente a través de la intervención de las noticias del ABC.

En este terreno es posible especular, por un lado, que los diarios tienen mayor efecto que la televisión, dadas su profundidad y la extensión que se otorga al tratamiento de la información. Una segunda alternativa es pensar que los políticos se sienten más expuestos ante los periódicos que ante los programas televisivos (Fuchs y Pfetsch, 1996). Lo cierto es que, más allá del soporte utilizado, se requiere de un alto nivel de congruencia entre las agendas informativas para que estas se instalen en el ámbito político; un comportamiento que desarrollaremos a profundidad en los próximos apartados (Eilders, 1997, 2000). En el mismo sentido, la espectacularización de la información suele contribuir a un mayor poder de agenda. Cuando los eventos espectaculares están ausentes, los medios no pueden actuar uniformemente. En ese caso, la clase de medio juega un rol: un medio fiable y respetado tiene un mayor impacto que uno marginal y ambiguo (Bartels, 1996).

¿Cómo se incorporan las redes sociales a los estudios de agenda building? Los trabajos que se incluyen aquí proponen una pregunta de investigación diferente: no se trata de explorar los efectos mediáticos en la agenda política sino de preguntarse por los cambios que genera una red social - Twitter, por caso- en las coberturas mediáticas, considerando que los periodistas son asiduos usuarios de este microblog en sus rutinas de trabajo (Lasorsa, Lewis y Holton, 2012; Parmelee y Bichard, 2012). Frente a la pregunta ¿cuán eficaces son los tuits de los políticos en sus efectos de agenda?, Parmelee (2014) concluye que las cuentas de los líderes políticos, de blogueros políticos y de think tanks o grupos de interés, lideran el proceso de agenda building, no solo por ser promotores de noticias, sino porque aportan ideas que permiten contextualizar y, eventualmente, corroborar datos. Coherente con la hipótesis de Parmelee, Calvo (2015) afirma que las redes sociales son entidades jerárquicas "dominadas por un número relativamente pequeño de actores” (p. 45) y, como tales, tienden a consolidar el estatus social de ciertas figuras políticas a partir de la diseminación de información que, finalmente, el público aprueba, acompaña y ayuda a difundir.

\section{Los temas}

Aunque no se ha sistematizado aún una tipología de los temas que contribuyen a generar un mayor efecto mediático sobre la política, Walgrave y van Aelst (2006) proponen una serie de rasgos que tendrían un impacto diferencial en esta relación. La 
cobertura noticiosa de los temas no experienciales - es decir, aquellos que no afectan directamente al público- puede tener mayor influencia en la agenda política. De hecho, cuando los medios son la única fuente de conocimiento de determinados issues, su impacto crece, tanto sobre el público como sobre los políticos (Lang y Lang, 1981). La determinación de responsabilidades alrededor de los asuntos es otro factor sensible: cuenta más la clara identificación de un actor político y de su involucramiento en un tema, en tanto que lo llama a actuar sobre este, que una atribución de responsabilidad ambigua (Pritchard, 1992).

También la novedad importa aquí. La atención de la clase política sobre asuntos novedosos aumenta dado que no le son familiares, no tienen una posición tomada al respecto y, sobre todo, no cuentan con la información respectiva suficiente (Brosius y Kepplinger, 1992). En efecto, la dramaticidad, la negatividad y la definición precisa del asunto como problemático son elementos que estimulan la atención de los políticos hacia las coberturas. Los temas sensacionales, caracterizados por una baja complejidad y la aparición de eventos dramáticos, permiten a los medios liderar la agenda (Soroka, 2002; Walgrave, Soroka y Nuytemans, 2007). La complejidad, en cambio, constriñe seriamente el potencial del efecto mediático, tanto en el público como en los políticos (Soroka et al., 2012). En consecuencia, suele ser ignorada en favor de una narrativa más convincente y definitiva.

\section{Los tiempos electorales y no electorales}

¿Qué ocurre en tiempos electorales? ¿Son los partidos políticos los que determinan la agenda noticiosa, o se subordinan al liderazgo mediático? Diversos estudios enfocados en períodos de campaña confirman el limitado —o a veces ausente - impacto de los medios sobre la agenda política (Brandenburg, 2002, 2004). En su trabajo Who follows whom, Brandenburg (2002) estudia la relación que hay entre los actores políticos británicos y el tipo de cobertura mediática que obtuvo la campaña de las elecciones generales de 1997, en
Gran Bretaña. Además, demuestra que tal vínculo entre medios y partidos políticos no es recíproco, sino que los partidos Conservador y Laborista, los más grandes, tienen efectos sobre la atención que los medios electrónicos prestan a determinados issues. En tanto, ni la televisión ni los medios gráficos parecen influir en la decisión de las agendas de los partidos políticos. Años después, con ocasión de la campaña electoral irlandesa, Brandenburg (2004) confirmará nuevamente que el partido más importante de ese país, el Fianna Fail ${ }^{2}$, es el mayor moldeador de la agenda mediática, fiel seguidora de las prioridades impuestas por la política.

Semetko y Canel (1997) analizan la relación que la televisión pública española TVE 1 y el canal privado Antena 3 mantuvieron con el poder político durante la campaña que se desplegó para los comicios presidenciales de España en 1996. Mediante observación de las salas de prensa, entrevistas y lecturas de documentos y estatutos - herramientas propias de la sociología de las redacciones-, estas investigadoras sistematizaron las diferencias que presentaban ambos canales en términos de las reglas y regulaciones bajo las cuales operaban. Retomamos las que consideramos más representativas. TVE 1 tiene por estatuto proveer imparcialidad y ecuanimidad en sus reportes, un aspecto que en teoría queda confinado a la omisión de cualquier tipo de opinión por parte del conductor. En contraste, en Antena 3 no hay ningún precepto que llame a la pluralidad de miradas. Además, el conductor participa activamente de la producción de la información y tiene control editorial sobre los contenidos. Pero la principal divergencia encontrada en la cobertura de la campaña está dada por la selección y el estilo de las noticias que hacen ambos competidores. TVE 1 es un "canal de transmisión de propaganda política [...] ¡Llaman a los partidos para preguntarles qué quieren cubrir ese día! Y lo hacen en nombre de una radiodifusión de servicio público" (Semetko y Canel, 1997, p. 475). ${ }^{3}$ Frente al poco margen o la falta de autonomía respecto del mensaje político encontrados en el caso del canal estatal, Antena 3 presenta un tipo de cobertura más analítica, evaluativa y autónoma. En otras palabras, frente a un 
canal público que es percibido por la audiencia como progubernamental, Antena 3 es visto como un canal antigubernamental, concluyen las autoras (Semetko y Canel, 1997, p. 477).

El grado de efecto de los medios en tiempos de campaña es más limitado debido a los vigorosos intentos de los políticos de ser ellos quienes fijen las prioridades del público. "Todo su esfuerzo está puesto en dominar el debate público” (Walgrave y van Aelst, 2006, p. 97). ${ }^{4}$ Para Eilders (2000), los políticos son afectados por los medios de la misma forma que los ciudadanos comunes, pues dependen de las pistas mediáticas para priorizar la información. Lo cierto es que los actores políticos no reaccionan ante la cobertura mediática en sí misma, sino ante el supuesto efecto que esta tiene sobre la opinión pública. Si se da por sentado que existe una asociación entre medios y opinión pública, una reacción natural de los políticos sería adoptar los temas cubiertos y seguir a los medios. Un mecanismo propio del priming, afirman Iyengar y Simon (1993), es que los políticos sean evaluados públicamente sobre la base de los temas priorizados por los medios. Pero la ausencia de una teoría que explique la dirección que toma el efecto de la agenda, pone en evidencia una carencia adicional: la inexistencia de un paraguas conceptual que diferencie entre la conducta de los actores políticos hacia los medios y el modelo cognitivo del establecimiento de la agenda pública. Mientras que el público tiene una relación inconsciente con la información noticiosa, los actores políticos deciden conscientemente, después de una reflexión estratégica, a qué prestar atención y a qué no. En tanto que el efecto sobre el público es cognitivo, la influencia sobre los políticos forma parte de un proceso conductual (Walgrave y van Aelst, 2006).

\section{¿Cómo ingresan los actores sociales a la agenda mediática?}

El estudio de los factores que inciden en la construcción de la agenda mediática tiene una larga tradición, incluso anterior a la Teoría de la Agenda Setting. Entre esos componentes se le dedicó una intensa atención a la relación que hay entre medios y política; una actitud que, sin embargo, no se sostuvo al momento de analizar la capacidad de otros actores sociales - fundamentalmente, los movimientos de protesta- de atraer la atención de los medios para instalar sus reivindicaciones.

Smith, McCarthy, McPhail y Augustyn (2001) estudian dos aspectos de este tenso vínculo: por una parte, indagan por la medida en que estos movimientos logran hacerse a un estado público por medio de la prensa y, por otra, si la cobertura mediática de sus acciones pone el foco en las problemáticas que ellos procuran instalar en la opinión pública, o si se concentra solo en sus protestas. Smith y su equipo observan que las protestas públicas no son una forma efectiva de comunicar los mensajes de los movimientos sociales. La selección de los acontecimientos que merecen ser cubiertos responde a criterios de noticiabilidad que son independientes de $-\mathrm{y}$ hasta contradicen- las características propias de las protestas sociales. De ahí que el nivel de discrepancia se acentúe en la medida en que los movimientos desafían directamente el sistema económico del que dependen las corporaciones mediáticas. Con este tenso vínculo de fondo, es posible pensar que la atención mediática que logren los dirigentes sociales tendrá un sesgo informativo, tanto en la instancia de la selección como en la de la descripción de los eventos.

Gitlin (1986) estudia el vínculo que hay entre el deseo de atención noticiosa que buscan los movimientos sociales y el tipo de cobertura resultante de estos. El autor sistematiza el tratamiento noticioso que The New York Times y los informativos de CBS realizaron del SDS. ${ }^{5}$ Cuando el SDS se convirtió en la gran noticia, los recursos que estructuraron el discurso mediático fueron la trivialización, la polarización, el énfasis en el disenso interno, la marginalización y el menosprecio por la efectividad del movimiento (Gitlin, 1986). Por ello, la cruzada de estos actores por instalar sus reivindicaciones en la agenda no puede reducirse a la cantidad de protestas o las formas inusuales de promoción de acciones colectivas. Deben considerarse los vínculos de 
tales iniciativas con los ciclos de la noticia (Smith et al., 2001). Para ser periodísticamente atractivas, sus reivindicaciones suelen ser presentadas de una forma dramática y polémica, mediante episodios controvertidos y situaciones de violencia (Boyle, Mccluskey, Devanathan, Stein y Mcleod, 2004). En tales casos, uno puede esperar que tal cobertura de la protesta mine el potencial impacto político de estos actores.

\section{El establecimiento de la agenda entre los medios}

Los medios de élite suelen ejercer una influencia sustancial sobre la agenda de los otros medios informativos. Aquello ocurre en los Estados Unidos con The New York Times (McCombs, 2006; Reese y Danielian, 1989). Es evidente el efecto de unos medios sobre otros, más precisamente, desde los de mayor tirada hacia los más pequeños (Breed, 1955). Pero, como veremos, tal fenómeno se da también en otras latitudes. Algunos de los estudios realizados en la Argentina evidenciaron dos mecanismos íntimamente relacionados: consonancia e influencia recíproca en la configuración de la agenda mediática, sobre todo en el caso de los matutinos de mayor circulación, Clarín y La Nación (Casermeiro de Pereson, 2004; Rey Lennon, 1998; Boczkowski, 2010).

A las semejanzas encontradas entre las coberturas de los distintos medios, que no solo publican los mismos tópicos sino que les dan un tratamiento similar, se las denomina "homogeneidad, consonancia o conformidad” (Donohue y Glasser, 1978; Riffe y Shaw, 1982, citados por Reese y Danielian, 1989). ${ }^{6}$ Es posible encontrar en Warren Breed (1955) uno de los antecedentes claves de los estudios de efecto de agenda entre los medios. Con el término proceso arterial, Breed grafica la influencia de los diarios líderes sobre los de menor tirada, con la consecuente estandarización o uniformidad de los contenidos mediáticos. La estandarización supone que varios periódicos contienen el mismo tema o similares, y que aquello es diseñado y organizado siguiendo el mismo modelo. Así pues, el principal ejemplo de estandarización es la tendencia de la prensa a presentar las mismas noticias en la parte alta de su tapa, excluyendo otras. El patrón de tal influencia, explica Breed, asume una forma arterial análoga a un modelo geológico ramificado por el cual los riachuelos, arroyos y las corrientes de agua derivan en un gran río, solo que en este caso el proceso es inverso porque el derrame se da desde la gran prensa hacia la menos influyente. Pero este efecto no solo se traduce en la disposición de las noticias en las páginas de un medio gráfico o en la ubicación y el tiempo dedicados a los distintos asuntos en un noticiero televisivo o radiofónico. La influencia a la que se refiere Breed deja sus marcas en la actitud política y la actividad profesional de los periodistas.

Más recientemente, Eilders operacionaliza el concepto de homogeneidad en su estudio sobre la relación que hay entre el sistema de medios y la agenda política, que resulta conveniente retomar en este apartado. Según esta investigadora, si los medios centran la atención en el mismo tema (focusing), lo encuadran de forma similar (consonance) y lo cubren con perseverancia (persistence), es posible esperar que haya un impacto notable sobre la agenda política (Eilders, 1997, 2000). La literatura define el término consonance como la dirección, el sentido de las opiniones existentes sobre un asunto. En cambio, el grado de correspondencia entre los temas —en términos de selección e ingreso a la agenda- es referido como centralidad (focusing). Si este tipo de evento, definido como evento focalizado (focusing event), no aparece explícitamente, puede bloquear el ascenso de un tema al juego de la política (Cobb y Elder, 1971).

Dentro de la tradición de la agenda setting, ya desde el estudio realizado en Chapel Hill en 1968 que dio luz a la este proceso, se puso en evidencia el alto grado de acuerdo en la cobertura de los temas por parte de The New York Times, Time, Newsweek, NBC y CBS. En aquella ocasión, McCombs y Shaw (1972) notaron, entre otros elementos, la existencia de una estructura 
organizacional estandarizada y de un mecanismo de socialización de las experiencias entre los reporteros. Lo que no advirtieron, como señalan Reese y Danielian (1989), es el liderazgo ejercido por ciertas organizaciones mediáticas como factor explicativo de tal consonancia.

Concretamente, The New York Times es considerado el árbitro final en cuanto a calidad y profesionalismo. A tal punto que "si el NYT no existiera, probablemente habría que inventarlo" (Gans, 2004, p. 181). . Según Gans, quien sugiere una historia espera prudentemente a que otro medio tome la iniciativa y, luego, vende esa idea argumentando que ha sido informada en otras partes. Esta homogeneidad puede tener consecuencias negativas para el funcionamiento de la democracia. En especial, para la diversidad de los contenidos noticiosos. "El peligro es la potencial influencia de un pequeño número de personas en la decisión de lo que millones de ciudadanos leerán” (Breed, 1955, p. 328). ${ }^{8}$

Los cambios producidos en el ecosistema de medios, fundamentalmente por el advenimiento de internet, se constituyeron en un desafío para las convenciones de influencia entre los medios que se fueron consolidando a lo largo de más de cuarenta años.

\section{La influencia de la propaganda política en la agenda mediática}

Frente a la imposibilidad de entrar en contacto directo con los candidatos presidenciales y conocer sus propuestas, los votantes suelen basar su decisión de voto en la información proveniente de los medios (Aruguete y Riorda, 2016). Es decir que sus discursos ejercen una influencia indirecta en la medida en que los medios seleccionan y difunden solo algunos temas contenidos en dichas declaraciones. Los candidatos juegan un rol activo en el proceso de agenda setting; intentan transmitir sus agendas a los votantes a través de los medios masivos (Bowers, 1977).

Los primeros intentos por comprender qué efectos, si los hubiere, generaba la propaganda política sobre los votantes, tuvieron lugar a comienzos de la década de 1970 en los Estados Unidos (Atkin, Bowen y Nayman, 1973). Por aquella época, Charlotte se constituyó en la primera oportunidad para analizar la propaganda televisiva desde la agenda setting. El propósito de aquel estudio, coordinado por Donald Shaw y Maxwell McCombs (1977), consistió —entre otros objetivos- en conocer cómo la prensa transmitía la agenda de los candidatos y qué aprendían los votantes de ella. Es decir, ¿̇en qué medida correspondían los temarios de los candidatos y los de los votantes? Para alcanzar esa meta, se monitorearon los spots del republicano Richard Nixon y los del demócrata George McGovern en tres cadenas de TV durante el prime time; se realizaron entrevistas a los votantes de Charlotte, y se analizó el contenido de diarios y noticieros televisivos durante la campaña. La correlación del ranking de las agendas de los candidatos y de los intereses de los votantes fue baja. Un nivel que, según Bowers, tiene varias explicaciones. Las indagaciones de los candidatos para conocer las preocupaciones del público se realizan al comienzo de la campaña, mientras que los issues que interesan a los votantes pueden variar hasta el día de los comicios. Además, los candidatos seleccionan los temas independientemente de los sondeos de opinión. "Usan la propaganda para generar interés sobre sus temas" (Bowers, 1977, p. 57 , las cursivas son del original). ${ }^{9}$

La tercera razón es de orden metodológico. Cuando a los votantes se les pregunta por los temas que les preocupan, es improbable que nombren las personalidades de los candidatos, un asunto que está en la cima de la agenda de estos políticos. De hecho, al sacar este aspecto del ranking, la correlación se vuelve algo más intensa. Aquella asociación no solo fue baja entre candidatos y votantes. Los temas predominantes en la agenda mediática tampoco se asociaron significativamente con los transmitidos en los spots. "La falta de correspondencia muestra las dificultades de los candidatos en sus intentos por transmitir sus agendas a través de los medios" (Bowers, 1977, p. 59).

Ahora bien, ¿la alta exposición a la televisión 
supone una mayor propensión a recordar los contenidos de los spots de campaña? Sí. Quienes encontraron correspondencia entre las distintas agendas fueron Roberts y McCombs (1994), casi veinte años después. En dos períodos distintos, estos autores analizaron los contenidos de las coberturas periodística y televisiva, al igual que de la propaganda de los candidatos, durante la campaña a la gobernación de Texas de 1990. A continuación, aplicaron un estudio de correlación entre ambos momentos (cross-lagged) para comprobar si existía algún vínculo de influencia entre la agenda de los medios y la de la campaña respecto de los temas más destacados. Los investigadores no solo encontraron que la agenda de los diarios ejercía un efecto significativo en los contenidos televisivos, sino que el diario de Austin y los tres canales de televisión locales reflejaron los temas priorizados por los candidatos. Es decir que, en efecto, las campañas políticas establecieron las agendas de los medios en aquella ocasión. La influencia del diario sobre los canales locales no era una sorpresa. Sí lo era la evidencia de que la propaganda política fuera un agenda setter, tanto de la televisión como de la prensa local. Aquello no supone afirmar taxativamente que los periodistas se vean influidos de manera directa por la agenda de la propaganda política, pero sí que responden a la agenda de la campaña como un todo. Los periodistas “están permanentemente expuestos a los tópicos clave de la campaña y es probable que sus reportes se vean influidos por dicho comportamiento" (Roberts y McCombs, 1994, p. 258).

\section{Reflexiones finales}

El propósito de este artículo ha sido describir los factores que participan en el proceso de construcción de la agenda mediática, desde una revisión bibliográfica de las dinámicas que tienen lugar en las capas de la cebolla (McCombs, 1992). En estas palabras finales, problematizamos algunos de esos elementos.

La dimensión más destacada de este review es la relación que se teje entre los medios y las fuentes de información, asumiendo que no todas las fuentes son iguales ni todas igualmente importantes y que, en consecuencia, el acceso a ellas por parte de los periodistas y viceversa no se distribuye de manera uniforme. Usamos el términofuentes de información en un sentido amplio, para referirnos a los actores que pujan por acceder a la agenda mediática e instalar allí sus reivindicaciones y propuestas.

Para ello nos servimos de una serie de estudios que se han desarrollado sobre la influencia de los agenda setters en las coberturas mediáticas $\mathrm{y}$, de manera complementaria, del efecto mediático sobre la agenda política. A la indagación por la relación que surge entre los decisores de políticas y la lógica de las rutinas periodísticas, se suma una dimensión que no había sido trabajada de manera sistemática en los estudios de agenda building: el acceso de los movimientos sociales a las coberturas noticiosas. ¿Por qué importa ponerlo de relieve aquí? Porque pone de manifiesto la correlación de fuerzas que se pone en juego en la interacción existente entre distintos actores y el sistema de medios.

A partir de esta complementación de perspectivas surgen algunas reflexiones. Por un lado, la visibilidad de determinados eventos no depende de la decisión unilateral que tomen los periodistas y editores, sino que remite a un campo de interés estructurado en el cual se da una puja intensa por imponer una definición de la realidad. En efecto, el reparto de los recursos de poder nunca es equitativo, en cuanto el tema propuesto por uno o varios actores y su capacidad de alcanzar estatus público no es independiente de las dinámicas de dicho campo. Se toma en cuenta entonces que la prensa no opera con autonomía del sistema político, de allí que el tipo de cobertura que prevalezca y sus decisiones con respecto a los niveles de noticiabilidad que alcance un asunto determinado, deban ser vistos en relación con el desarrollo político en el cual esta es uno de los motores. "La evaluación de la noticiabilidad es un fenómeno negociado, constituido por las actividades de una compleja burocracia diseñada para supervisar la red informativa" (Tuchman, 1983, p. 51). ${ }^{10}$ Por ello, restringir su papel a la función primaria 
de diseminador de información es subestimar su legítimo rol activo en el proceso de formación de la opinión (Eilders, 2000).

En sintonía con esta mirada que se tiende sobre la noticiabilidad como fenómeno negociado, cabe insistir en las dificultades que tienen los actores menos poderosos para acceder a la agenda mediática en condiciones noticiosas de mayor estabilidad y cuidado. Un ejemplo notable es la capacidad de los movimientos sociales de plasmar sus reivindicaciones en las coberturas. Sus agendas son distintas de las agendas noticiosas, porque la propia lógica productiva de los medios va en contra del acceso de los dirigentes sociales a sus páginas y pantallas. Cuando ingresan lo hacen a costa de neutralizar y socavar sus reclamos y premisas (Smith et al., 2001, p. 1398). La lógica organizacional y las rutinas productivas obligan a las organizaciones sociales a justificar por qué consideran que ciertos temas deberían recibir atención por parte de los medios (Aruguete, 2011).

Uno de los intereses centrales de los apartados mencionados ha sido indagar por cómo se miden los grados de influencia que ejercen distintos actores. En este punto, Charron (1998) plantea una inquietud significativa con respecto a las dificultades de la agenda building para observar la influencia de los que deciden en el proceso de formación de la agenda de los asuntos públicos. Coincidimos con este autor en que la mera cuantificación de lo que define como la agenda de los quienes, no da cuenta del verdadero ejercicio de poder que ejercen las fuentes, ni de su capacidad de imponer o condicionar cierta definición de la realidad. En este punto, se vuelve necesario incorporar la dimensión económico-financiera de la estructura de los medios que se suma al eje político-cultural, para tener una comprensión más compleja y acabada de su capacidad de fijar la agenda pública. Tal inclusión se justifica, sobre todo, porque este es un aspecto para nada soslayado por los líderes políticos y actores corporativos a la hora de considerar qué temas de los priorizados en las noticias integran a sus propias agendas y en qué tipo de coberturas participan como fuentes.
Ahora bien, no todas las noticias tienen el mismo poder para influir en la agenda política. ¿Existen diferencias entre los medios a la hora de fijar los temas que preocuparán a los líderes políticos? Ya hemos observado que para que un tema genere efectos significativos sobre la arena política, es necesario alcanzar un alto nivel de congruencia entre las coberturas informativas, esto es: centrar la atención en el mismo tema, encuadrarlo de forma similar y cubrirlo con perseverancia (Eilders, 1997). El sistema de medios, afirma Eilders, actúa de manera consonante solo bajo ciertas circunstancias, en su propósito de diferenciarse de sus competidores; y el foco puesto en determinados issues los identifica editorialmente: los distingue. En este punto, la dificultad reside en la ausencia de una tradición académica que haya alcanzado un consenso generalizado y que permita comprender tal nivel de consonancia, si lo hubiere.

Llegados a este punto, advertimos la necesidad de analizar de manera crítica la trasferencia de relevancia de una agenda a otra (McCombs, 2010). La relación de los medios con la opinión pública no es asimilable al diálogo tenso que ambos mantienen con los líderes políticos, ya que no reaccionan de la misma forma ante la agenda noticiosa (Walgrave y van Aelst, 2006). De hecho, el poder que tienen los medios de instalar una agenda reposa, no tanto en su influencia directa sobre un público generalizado, sino en la percepción que los expertos y los decisores de políticas tienen sobre tal efecto mediático en la opinión pública.

En definitiva, el discurso mediático busca que sus opiniones tengan resonancia tanto en las audiencias como en la política, de ahí que en el proceso de su construcción quede manifiesta la influencia recíproca de estos tres ámbitos, al menos en algunos aspectos. Mediante la selección y el énfasis en ciertos temas, los medios expresan su particular posición política desde un perfil ideológico que los distingue (Eilders, 2000). A diferencia de esta autora, notamos un fuerte nivel de consonancia al interior del sistema mediático; una lógica de comportamiento estructural que no se ha modificado desde que fuera advertida por 
Breed a mediados del siglo pasado. La estandarización (Breed, 1955) — la selección y jerarquización de una serie de temas de manera generalizada - se da tanto en los medios tradicionales como en los nuevos medios y las redes sociales. Al respecto, cabe destacar que el grado de concentración en la oferta de contenidos en internet es mayor que el de los medios de élite. "Los mercados de medios siempre estuvieron muy concentrados por razones económicas que están bien estudiadas, pero el mercado online es el más concentrado de todos: hay menos de una decena de grandes jugadores" (Schuliaquer, 2014). ${ }^{11}$

¿Por qué es tan fuerte este impulso de los medios a confirmar con otros el juicio periodístico sobre las noticias? Porque la adherencia a los canales rutinarios y la semejanza de las historias permiten a los reporteros probarse a sí mismos que conocen la noticia real. "Consistencia es igual a precisión” (Reese y Danielian, 1989, p. 34). Demasiada semejanza entre los contenidos mediáticos entra en conflicto con el valor clave de la pluralidad — un conjunto de miradas y voces diversas - que debe alcanzar una sociedad democrática. Más grave aún es cuando la mayor disponibilidad de canales de información para la audiencia y las mejores posibilidades de cobertura ofrecidas por parte de los medios no garantizan una diversificación de los contenidos. "Es posible que haya más trabajadores de prensa ahora que hace quince años, pero harán poco por lograr una mayor diversidad si sólo cubren los grandes temas" (Reese y Danielian, 1989, p. 31).

\section{Referencias}

Aruguete, N. (2011). Los medios y la privatización de Entel. Berlín: Editorial Académica Española.

Aruguete, N. (2013). La narración del espectáculo político: pensar la relación entre sistema de medios y poder político. Austral Comunicación, 2(1), 205-216.

Aruguete, N. (2015). El poder de la agenda. Política, medios y público. Buenos Aires: Biblos.
Aruguete, N. y Riorda, M. (2016). Una imagen vale más que mil políticas: la estrategia discursiva de las campañas electorales de los candidatos ganadores en América Latina. Communication \& Society 29(2), 173-192.

Atkin, C., Bowen, L. y Nayman, O. (1973). Quality versus quantity in televised political ads. Public Opinion Quarterly, 37(2), 209-224.

Bartels, L. M. (1996). Politicians and the press: Who leads, who follows? En Annual Meeting of the American Political Science Association (pp. 1-60). San Francisco: American Political Science Association.

Baumgartner, F., Bryan, D. y Leech, B. (1997). Media attention and congressional agendas. En S. Iyengar y R. Reeves (eds.), Do the Media Govern?: Politicians, Voters, and Reporters in America (pp. 349-363). Thousand Oak: Sage.

Boczkowsky, P. (2010). News at Work. Imitation in an Age of Information Abundance. Londres: The University of Chicago Press.

Bowers, T. A. (1977). Candidate advertising: The agenda is the message. En Shaw, D. (ed.), The Emergence of American Political Issues: The agenda setting function of the press (pp. 53-67). St. Paul: West.

Boyle, M. P., Mccluskey, M. R., Devanathan, N., Stein, S. E. y Mcleod, D. (2004). The Influence of Level of Deviance and Protest Type on Coverage of Social Protest in Wisconsin from 1960 to 1999. Mass Communication and Society, 7(1), 43-60.

Brandenburg, H. (2002). Who follows whom? The Impact of Parties on Media Agenda Formation in the 1997 British General Election Campaign. Pess/Politics, 7(3), 34-54.

Brandenburg, H. (2004). Communicating issue salience a comparative study into campaign effects on media agenda formation. En EPOP 2004 Annual Conference (p. 42). Oxford, UK: EPOP.

Breed, W. (1955). Newspaper "Opinion leaders" and Processes of Standardization. Journalism \& Mass Communication Quarterly, 32(3), 277-328. 
Brosius, H. y Kepplinger, M. (1992). Beyond Agenda Setting: The Influence of Partisanship and Television Reporting on the Electorate's Voting Intentions. Journalism Quarterly, 69(4), 893-901.

Calvo, E. (2015). Anatomía política de Twitter en Argentina. Buenos Aires: Capital Intelectual.

Casermeiro de Pereson, A. (2004). Los medios en las elecciones: la agenda setting en la Ciudad de Buenos Aires. Buenos Aires: Educa.

Cervantes Barba, C. (2001). La sociología de las noticias y el enfoque agenda setting. Convergencia, 24, 49-65.

Charron, J. (1998). Los medios y las fuentes. Los límites al modelo del agenda setting. En G. Gauthier, A. Gosselin y J. Mouchon (eds.), Comunicación y política (pp. 72-93). Barcelona: Gedisa.

Cobb, R. y Elder, C. (1971). The Politics of Agenda Building: An Alternative Perspective for Modern Democratic Theory. The Journal of Politics, 33(4), 892-915.

Cohen, B. (1993). The Press and Foreign Policy. Connecticut: University of California.

Eilders, C. (1997). The impact of editorial content on the political agenda in Germany: theoretical assumptions and open questions regarding a neglected subject in mass communication research. WZB. Berlin Social Science Center. Discussion Paper, FS III, 97-102.

Eilders, C. (2000). Media as Political Actors? Issue Focusing and Selective Emphasis in the German Quality Press. German Politics, 9(3), 181-206.

Fuchs, D. y Pfetsch, B. (1996). Die beobachtung der ofentlichen meinung durch das regierungssystem. En W. van den Daele y F. Neidhardt (eds.), Kommunikation und Entscheidung. Politische Funktionen öffentlicher Meinungsbildun (pp. 103-138). Berlín: Gebundene Ausgabe.

Gans, H. (2004). Deciding What's News. A study CBS Evening News, NBC Nightly News, Newsweek, and Time. Evanston: Random House.
Gilberg, S., Eyal, C., McCombs, M. E. y Nicholas, D. (1991). The State of the Union Address and the Press Agenda. En D. Protess y M. E. McCombs (eds.), Agenda setting. Reading on media, Public Opinion and Policymaking (pp. 223-236). Nueva York: Lawrence Erlbaum.

Gitlin, T. (1986). Convertir a los movimientos de protesta en temas periodísticos. En D. Graber (ed.), El poder de los medios en la política (pp. 289-302). Buenos Aires: GEL.

Golding, P. (1981). The Missing Dimensions: News Media and the Management of Social Change. En E. Katz y T. Szecsko (eds.), Mass Media and Social Change (pp. 63-81). Beverly Hills: Sage.

Herman, E. (1993). The Media's Role in U.S. Foreign Policy. Journal of International Affairs, 47(1), 23-46.

Iyengar, S. y Simon, A. (1993). News Coverage of the Gulf Crisis and Public Opinion. A Study of Agenda-Setting, Priming and Framing. Communication Research, 20(3), 365-383.

Kunczik, M. (1991), Conceptos del periodismo: Norte y Sur. Bonn: Friedrich-Ebert-Stiftung.

Lang, G. y Lang, K. (1981). Watergate: An Exploration of the Agenda-Building Process. En G. Cleveland Wilhoit (ed.), Mass Communication Review Yearbook (pp. 447-468). Newbury Park: Sage.

Lasorsa, D. L., Lewis, S. C. y Holton, A. E. (2012). Normalizing Twitter: Journalism Practice in an Emerging Space. Journalism Studies, 13(1), 19-36.

Livingston, S. (1997). Beyond the "CNN-Effect": The Media-Foreign Policy Dynamic. En P. Norris (ed.), Politics and the Press: The news media and their influences (pp. 291-314). Boulder: Lynne Rienner.

McCombs, M. E. (2006). Estableciendo la agenda. Barcelona: Paidós.

McCombs, M. E. (2010). Extending our theoretical maps: Psychology of agenda setting. Central European Journal of Communication, 2, 197-206. 
McCombs, M. E. (1992). Explorers and Surveyors: Expanding Strategies for Agenda-Setting Research. Journalism \& Mass Communication Quarterly, 69(4), 813-824.

McCombs, M. E. y Shaw, D. (1972). The Agenda Setting Function of the Mass Media. Public Opinion Quarterly, 36, 176-187.

Mermin, J. (1997). Television News and the American Intervention in Somalia: The Myth of a Media-Driven Foreign Policy. Political Science Quarterly, 112, 385-404.

Parmelee, J. y Bichard, S. (2012). Politics and the Twitter Revolution: How Tweets Influence the Relationship Between Political Leaders and the Public. Lanham: Lexington Books.

Parmelee, J. H. (2014). The agenda building function of political tweets. New Media $\mathcal{E}$ Society, 16(3), 434-450.

Pritchard, D. (1992). The news media and public policy agendas. En D. Kennamer (ed.), Public Opinion, The Press, and Public Policy. An Introduction (pp. 103-112). Westpoint: Praeger.

Reese, S. y Danielian, L. (1989). Inter-Media Influence and the Drug Issue: Converging on Cocaine. Communication Campaigns about Drugs: Government, Media, and the Public, 5, 29-46.

Rey Lennon, F. (1998). Argentina: elecciones 1997. Los diarios nacionales y la campaña electoral. Buenos Aires: Freedom Forum-Universidad Austral.

Roberts, M. S. y McCombs, M. E. (1994). Agenda setting and political advertising: Origins of the news agenda. Political Communication, 11, 249-262.

Sádaba, T. (2008). Framing: el encuadre de las noticias. El binomio terrorismo-medios. Buenos Aires: La Crujía.

Schlesinger, P. (1992). Repensando la sociología del periodismo: estrategias de las fuentes y límites del centralismo en los medios. Estudios sobre las culturas contemporáneas, 4(14), 279-307.

Schuliaquer, I. (2014). El poder de los medios. Buenos Aires: Capital Intelectual.

Semetko, H. y Canel, M. J. (1997). AgendaSenders versus Agenda-Setters: Television in Spain’s 1996 Election Campaign. Political
Communication, 14(4), 459-479.

Shaw, D. L. y McCombs, M. E. (eds.) (1977). The Emergence of American Political Issues. The Agenda-Setting Function of the Press. St. Paul: West.

Shoemaker, P. y Reese, S. (1996). Mediating the Message. Theories of Influences on Mass Media Content. Nueva York: Longman.

Sigal, L. V. (1973). Reporters and Officials: The Organization and Politics of Newsmaking. Lexington: D. C. Heath.

Smith, J., McCarthy, J., McPhail, C. y Augustyn, B. (2001). From Protest to Agenda Building: Description Bias in Media Coverage. Social Forces, 79(4), 1897-1423.

Soroka, S. (2002). Issue Attributes and AgendaSetting by Media, the Public and Policymakers in Canada. International Journal of Public Opinion Research, 14(3), 264-285.

Soroka, S., Lawlor, A., Farnsworth, S. y Young, L. (2012). Mass Media and Policymaking. En E. Araral, S. Fritzen, M. Howlett, M. Ramesh y X. Wu (eds.). Routledge Handbook of Public Policy (pp. 1-15). Nueva York: Routledge.

Trumbo, C. (1996). Constructing Climate Change: Claims and Frames in US News Coverage of an Environmental Issue. Public Understanding of Science, 5, 269-283.

Tuchman, G. (1972). Objectivity as Strategic Ritual: an Examination of Newsmen's Notions of Objectivity. American Journal of Sociology, 77(4), 660-679.

Tuchman, G. (1983). La producción de las noticias. Estudio sobre la construcción de la realidad. Buenos Aires: Ediciones Gustavo Gili.

Walgrave, S. y van Aelst, P. (2006). The Contingency of the Mass Media's Political Agenda Setting Power: Toward a Preliminary Theory. Journal of Communication, 56(1), 88-109.

Walgrave, S., Soroka, S. y Nuytemans, M. (2007). The Mass Media's Political Agenda-Setting Power: A Longitudinal Analysis of Media, Parliament, and Government in Belgium (1993 to 2000). Comparative Political Studies, 41(6), 814-836. 
Wanta, W. (1997). The Public and National Agenda: How people learn about important issues. Mahwah: Lawrence Erlbaum.

Wanta, W., Stephenson, M. A., Vanslyke Turk, J. y McCombs, M. E. (1989). How President's State of Union Talk Influenced News Media Agendas. Journalism Quarterly, 66, 537-541.

Weaver, D. y Gray, R. (1982). Media agenda setting and media manipulation. Mass Communication Review Yearbook, 3, 537-554.

White, D. (1950). The "Gate-Keeper": A Case Study in the Selection of News. Journalism Quarterly, 34, 383-390.

Wright, C. R. (1959). Mass Communication: A sociological perspective. Nueva York: Random House.

\section{Notas}

1. El texto transcrito, al igual que otros fragmentos sucesivos que aludan a esta referencia, corresponde a una traducción de la autora.

2. Fianna Fail es el Partido Republicano de Irlanda, cuya expresión en irlandés significa soldados del destino.

3. El texto transcrito, al igual que otros fragmentos sucesivos que aludan a esta referencia, corresponde a una traducción de la autora.

4. El texto transcrito, al igual que otros fragmentos sucesivos que aludan a esta referencia, corresponde a una traducción de la autora.

5. El Students for a Democratic Society (SDS) fue un importante movimiento de protesta de la Nueva Izquierda en los ámbitos universitarios estadounidenses en la década de 1960.

6. El texto transcrito, al igual que otros fragmentos sucesivos que aludan a esta referencia, corresponde a una traducción de la autora.

7. El texto transcrito, al igual que otros fragmentos sucesivos que aludan a esta referencia, corresponde a una traducción de la autora.

8. El texto transcrito, al igual que otros fragmentos sucesivos que aludan a esta referencia, corresponde a una traducción de la autora.

9. El texto transcrito, al igual que otros fragmentos sucesivos que aludan a esta referencia, corresponde a una traducción de la autora.

10. El texto transcrito, al igual que otros fragmentos sucesivos que aludan a esta referencia, corresponde a una traducción de la autora.

11. Entrevista realizada al investigador Pablo Boczkowski.
Cómo citar este artículo

Aruguete, N. (2017). Agenda building. Revisión de la literatura sobre el proceso de construcción de la agenda mediática. Signo y Pensamiento, 36(70), 36-52. https://doi. org/ 10.11144/Javeriana.syp36-70.abrl 
